Commun. Korean Math. Soc. 29 (2014), No. 1, pp. 109-121

http://dx.doi.org/10.4134/CKMS.2014.29.1.109

\title{
SPECIAL HALF LIGHTLIKE SUBMANIFOLDS OF AN INDEFINITE SASAKIAN MANIFOLD
}

\author{
DAE Ho JiN
}

\begin{abstract}
In this paper, we study the geometry of half lightlike submanifolds of an indefinite Sasakian manifold. There are several different types of half lightlike submanifolds of an indefinite Sasakian manifold according to the form of its structure vector field. We study two types of them here: tangential and ascreen half lightlike submanifolds.
\end{abstract}

\section{Introduction}

The class of codimension 2 lightlike submanifolds of semi-Riemannian manifolds is compose of two classes by virtue of the rank of its radical distribution, which are called half lightlike submanifold or coisotropic submanifold [5]. Half lightlike submanifold is a particular case of $r$-lightlike submanifold [4] such that $r=1$ and its geometry is more general form than that of coisotrophic submanifolds or lightlike hypersurfaces. Much of the works on half lightlike submanifolds will be immediately generalized in a formal way to arbitrary $r$ lightlike submanifolds. Recently many authors have studied the geometry of lightlike submanifolds of indefinite Sasakian manifolds [7, 8, 9, 12].

In this paper, we study two types of half lightlike submanifolds of an indefinite Sasakian manifold, named by tangential and ascreen half lightlike submanifolds. In Section 3, we prove three characterization theorems for tangential half lightlike submanifolds: (1) There exists no screen conformal tangential half lightlike submanifold of an indefinite Sasakian manifold. (2) There exists no tangential half lightlike submanifold of an indefinite Sasakian manifold such that its screen distribution is totally umbilical. (3) There exists no totally umbilical tangential half lightlike submanifold of an indefinite Sasakian manifold.

In Section 4, we prove three characterization theorems for ascreen half lightlike submanifolds: (1) There exists no screen conformal ascreen half lightlike submanifold of an indefinite Sasakian manifold. (2) There exists no ascreen half

Received April 20, 2012.

2010 Mathematics Subject Classification. Primary 53C25, 53C40, 53C50.

Key words and phrases. tangential and ascreen half lightlike submanifold, totally umbilical, screen conformal, indefinite Sasakian manifold. 
lightlike submanifold of an indefinite Sasakian manifold such that its screen distribution is totally umbilical. (3) There exists no proper totally umbilical ascreen half lightlike submanifold of an indefinite Sasakian manifold. In addition to these main theorems, we study the geometry of totally umbilical ascreen half lightlike submanifolds of an indefinite Sasakian manifold.

\section{Half lightlike submanifolds}

An odd dimensional semi-Riemannian manifold $(\bar{M}, \bar{g})$ is called an indefinite Sasakian manifold $[7,8,12]$ if there exists an indefinite almost contact metric structure $(J, \theta, \zeta, \bar{g})$, where $J$ is a $(1,1)$-type tensor field, $\zeta$ a vector field which is called the structure vector field and $\theta$ a 1-form satisfying

$$
\left\{\begin{array}{c}
J^{2} X=-X+\theta(X) \zeta, J \zeta=0, \theta \circ J=0, \theta(\zeta)=1, \\
\bar{g}(\zeta, \zeta)=\varepsilon, \quad \bar{g}(J X, J Y)=\bar{g}(X, Y)-\varepsilon \theta(X) \theta(Y), \\
\theta(X)=\varepsilon \bar{g}(\zeta, X), \quad d \theta(X, Y)=\bar{g}(J X, Y), \varepsilon= \pm 1, \\
\bar{\nabla}_{X} \zeta=J X, \\
\left(\bar{\nabla}_{X} J\right) Y=\varepsilon \theta(Y) X-\bar{g}(X, Y) \zeta,
\end{array}\right.
$$

for any vector fields $X, Y$ on $\bar{M}$, where $\bar{\nabla}$ is the Levi-Civita connection of $\bar{M}$.

It is known [9] that, for any indefinite Sasakian manifolds $\bar{M}$, the structure vector field $\zeta$ of $\bar{M}$ is spacelike, that is, $\varepsilon=1$.

A submanifold $M$ of a semi-Riemannian manifold $\bar{M}$ of codimension 2 is called a half lightlike submanifold if the radical distribution $\operatorname{Rad}(T M)=T M \cap$ $T M^{\perp}$ of $M$ is a vector subbundle of the tangent bundle $T M$ and the normal bundle $T M^{\perp}$ of rank 1 . Therefore there exist complementary non-degenerate distributions $S(T M)$ and $S\left(T M^{\perp}\right)$ of $\operatorname{Rad}(T M)$ in $T M$ and $T M^{\perp}$, respectively, which are called the screen and co-screen distributions on $M$, such that

$$
T M=\operatorname{Rad}(T M) \oplus_{\text {orth }} S(T M), T M^{\perp}=\operatorname{Rad}(T M) \oplus_{\text {orth }} S\left(T M^{\perp}\right),
$$

where the symbol $\oplus_{\text {orth }}$ denotes the orthogonal direct sum. We denote such a half lightlike submanifold by $M=(M, g, S(T M))$. Denote by $F(M)$ the algebra of smooth functions on $M$ and by $\Gamma(E)$ the $F(M)$ module of smooth sections of a vector bundle $E$ over $M$. Choose $L \in \Gamma\left(S\left(T M^{\perp}\right)\right)$ as a unit vector field with $\bar{g}(L, L)=\epsilon(= \pm 1)$. Consider the orthogonal complementary distribution $S(T M)^{\perp}$ to $S(T M)$ in $T \bar{M}$. Certainly $\xi \in \Gamma(\operatorname{Rad}(T M))$ and $L$ belong to $S(T M)^{\perp}$. Hence we have the following orthogonal decomposition:

$$
S(T M)^{\perp}=S\left(T M^{\perp}\right) \oplus_{\text {orth }} S\left(T M^{\perp}\right)^{\perp},
$$

where $S\left(T M^{\perp}\right)^{\perp}$ is the orthogonal complementary to $S\left(T M^{\perp}\right)$ in $S(T M)^{\perp}$. For any null section $\xi$ of $\operatorname{Rad}(T M)$ on a coordinate neighborhood $\mathcal{U} \subset M$, there exists a uniquely defined null vector field $N \in \Gamma(\operatorname{lt}(T M))$ satisfying

$$
\bar{g}(\xi, N)=1, \bar{g}(N, N)=\bar{g}(N, X)=\bar{g}(N, L)=0, \forall X \in \Gamma(S(T M)) .
$$

We call $N, \operatorname{lt}(T M)$ and $\operatorname{tr}(T M)=S\left(T M^{\perp}\right) \oplus_{\text {orth }}$ ltr $(T M)$ the lightlike transversal vector field, lightlike transversal vector bundle and transversal vector 
bundle of $M$ with respect to $S(T M)$, respectively. Then the tangent bundle $T \bar{M}$ of the ambient manifold $\bar{M}$ is decomposed as follows:

$$
\begin{aligned}
T \bar{M} & =T M \oplus \operatorname{tr}(T M)=\{\operatorname{Rad}(T M) \oplus \operatorname{tr}(T M)\} \oplus_{\text {orth }} S(T M) \\
& =\{\operatorname{Rad}(T M) \oplus l \operatorname{tr}(T M)\} \oplus_{\text {orth }} S(T M) \oplus_{\text {orth }} S\left(T M^{\perp}\right) .
\end{aligned}
$$

Let $\bar{\nabla}$ be the Levi-Civita connection of $\bar{M}$ and $P$ the projection morphism of $T M$ on $S(T M)$ with respect to the decomposition (2.4). Then the local Gauss and Weingarten formulas for $M$ and $S(T M)$ are given, respectively, by

$$
\begin{aligned}
& \bar{\nabla}_{X} Y=\nabla_{X} Y+B(X, Y) N+D(X, Y) L, \\
& \bar{\nabla}_{X} N=-A_{N} X+\tau(X) N+\rho(X) L, \\
& \bar{\nabla}_{X} L=-A_{L} X+\phi(X) N ; \\
& \nabla_{X} P Y=\nabla_{X}^{*} P Y+C(X, P Y) \xi, \\
& \nabla_{X} \xi=-A_{\xi}^{*} X-\tau(X) \xi,
\end{aligned}
$$

for all $X, Y \in \Gamma(T M)$, where $\nabla$ and $\nabla^{*}$ are induced linear connections of $M$ and on $S(T M)$, respectively, $B$ and $D$ are called the local second fundamental forms of $M, C$ is called the local second fundamental form on $S(T M), A_{N}, A_{\xi}^{*}$ and $A_{L}$ are linear operators on $T M$ and $\tau, \rho$ and $\phi$ are 1-forms on $T M$.

Since $\bar{\nabla}$ is torsion-free, $\nabla$ is torsion-free, and $B$ and $D$ are symmetric. From the facts $B(X, Y)=\bar{g}\left(\bar{\nabla}_{X} Y, \xi\right)$ and $D(X, Y)=\epsilon \bar{g}\left(\bar{\nabla}_{X} Y, L\right)$, we know that $B$ and $D$ are independent of the choice of a screen distribution and satisfy

$$
B(X, \xi)=0, \quad D(X, \xi)=-\epsilon \phi(X), \quad \forall X \in \Gamma(T M) .
$$

The induced connection $\nabla$ of $M$ is not metric and satisfies

$$
\left(\nabla_{X} g\right)(Y, Z)=B(X, Y) \eta(Z)+B(X, Z) \eta(Y),
$$

for all $X, Y, Z \in \Gamma(T M)$, where $\eta$ is a 1 -form on $T M$ such that

$$
\eta(X)=\bar{g}(X, N), \forall X \in \Gamma(T M) .
$$

But the connection $\nabla^{*}$ on $S(T M)$ is metric. The above three local second fundamental forms are related to their shape operators by

$$
\begin{array}{lc}
B(X, Y)=g\left(A_{\xi}^{*} X, Y\right), & \bar{g}\left(A_{\xi}^{*} X, N\right)=0, \\
C(X, P Y)=g\left(A_{N} X, P Y\right), & \bar{g}\left(A_{N} X, N\right)=0, \\
\epsilon D(X, P Y)=g\left(A_{L} X, P Y\right), & \bar{g}\left(A_{L} X, N\right)=\epsilon \rho(X), \\
\epsilon D(X, Y)=g\left(A_{L} X, Y\right)-\phi(X) \eta(Y), \forall X, Y \in \Gamma(T M) .
\end{array}
$$

A half lightlike submanifold $M=(M, g, \nabla)$ equipped with a degenerate metric $g$ and a linear connection $\nabla$ is said to be of constant curvature $c$ if there exists a constant $c$ such that the curvature tensor $R$ of $\nabla$ satisfies

$$
R(X, Y) Z=c\{g(Y, Z) X-g(X, Z) Y\}, \quad \forall X, Y, Z \in \Gamma(T M) .
$$

To discuss the geometry of half lightlike submanifolds of an indefinite Sasakian manifold, we need the following results [11]: 
Theorem 2.1. Let $M$ be a half lightlike submanifold of an indefinite Sasakian manifold $\bar{M}$. Then the structure vector field $\zeta$ of $\bar{M}$ does not belong to the vector bundles $\operatorname{Rad}(T M), \operatorname{lt}(T M)$ and $S\left(T M^{\perp}\right)$.

Theorem 2.2. Let $M$ be a tangential half lightlike submanifold of an indefinite Sasakian manifold $\bar{M}$. Then the distributions $J(\operatorname{Rad}(T M)), J(\operatorname{lt}(T M))$ and $J\left(S\left(T M^{\perp}\right)\right)$ are vector subbundles of $S(T M)$ of rank 1.

\section{Tangential half lightlike submanifolds}

Definition. A half lightlike submanifold $M$ of an indefinite Sasakian manifold $\bar{M}$ is said to be tangential if the structure vector field $\zeta$ is tangent to $M$.

Definition. (1) A half lightlike sbumanifold $M$ of $\bar{M}$ is screen conformal [6] if there exists a non-vanishing smooth function $\varphi$ on a neighborhood $\mathcal{U}$ in $M$ such that $A_{N} X=\varphi A_{\xi}^{*} X$ for all $X \in \Gamma(T M)$, or equivalently,

$$
C(X, P Y)=\varphi B(X, Y), \quad \forall X, Y \in \Gamma(T M) \text {. }
$$

(2) A screen distribution $S(T M)$ of $M$ is totally umbilical [4] in $M$ if there exists a smooth function $\gamma$ on a neighborhood $\mathcal{U}$ in $M$ such that

$$
C(X, P Y)=\gamma g(X, Y), \quad \forall X, Y \in \Gamma(T M) .
$$

(3) A half lightlike sbumanifold $M$ of $\bar{M}$ is totally umbilical [4] if, on any coordinate neighborhood $\mathcal{U}$, there is a smooth vector field $\mathcal{H} \in \Gamma(\operatorname{tr}(T M))$, called the transversal curvature vector field of $M$, such that

$$
h(X, Y)=\mathcal{H} g(X, Y), \quad \forall X, Y \in \Gamma(T M),
$$

where $h(X, Y)=B(X, Y) N+D(X, Y) L$ is a second fundamental form tensor of $M$. In case $\mathcal{H}=0$ (or $\mathcal{H} \neq 0$ ) on $\mathcal{U}$, we say that $M$ is totally geodesic (or proper totally umbilical).

It is easy to see that $M$ is totally umbilical if and only if, on each coordinate neighborhood $\mathcal{U}$, there exist smooth functions $\beta$ and $\delta$ such that

$$
B(X, Y)=\beta g(X, Y), D(X, Y)=\delta g(X, Y), \forall X, Y \in \Gamma(T M) .
$$

Theorem 3.1. (1) There exists no screen conformal tangential half lightlike submanifold $M$ of an indefinite Sasakian manifold $\bar{M}$.

(2) There exists no tangential half lightlike submanifold $M$ of an indefinite Sasakian manifold $\bar{M}$ such that $S(T M)$ is totally umbilic in $M$.

(3) There exists no totally umbilical tangential half lightlike submanifold $M$ of an indefinite Sasakian manifold $\bar{M}$.

Proof. Applying $\bar{\nabla}_{X}$ to $\bar{g}(J \xi, N)=0$ and using (2.1), (2.3), (2.6), (2.7), (2.10), (2.11), (2.13) and (2.14), we have

$$
B(X, J N)=C(X, J \xi), \quad \forall X \in \Gamma(T M) .
$$

Since $\zeta$ is tangent to $M$, from the equations (2.2) and (2.6), we obtain

$$
J X=\nabla_{X} \zeta+B(X, \zeta) N+D(X, \zeta) L, \quad \forall X \in \Gamma(T M) .
$$


Taking the scalar product with $\xi$ in this equation, we have

$$
B(X, \zeta)=-g(X, J \xi), \quad \forall X \in \Gamma(T M) .
$$

Replacing $X$ by $J \xi$ and $J N$ in this equation by turns, we have

$$
B(J \xi, \zeta)=0, \quad B(J N, \zeta)=-1 .
$$

(1) If $M$ is screen conformal, then, using (3.1) and (3.2), we have

$$
-1=B(J N, \zeta)=C(J \xi, \zeta)=\varphi B(J \xi, \zeta)=\varphi 0=0 .
$$

It is a contradiction. Thus there exists no screen conformal tangential half lightlike submanifold $M$ of an indefinite Sasakian manifold $\bar{M}$.

(2) If $S(T M)$ is totally umbilical in $M$, then, from (3.1), we get

$$
B(X, J N)=\gamma g(X, J \xi), \forall X \in \Gamma(T M) .
$$

Using this and the second equation of $(3.2)$ [denote $(3.2)_{2}$ ], we have

$$
-1=B(J N, \zeta)=\gamma g(J \xi, \zeta)=\gamma \theta(J \xi)=\gamma 0=0 .
$$

It is also a contradiction. Thus there exists no tangential half lightlike submanifold $M$ of $\bar{M}$ such that $S(T M)$ is totally umbilical on $M$.

(3) If $M$ is totally umbilical, from the second equation of (3.2), we have

$$
-1=B(J N, \zeta)=\beta g(J N, \zeta)=\beta \theta(J N)=\beta 0=0 .
$$

It is a contradiction. Thus there exists no totally umbilical tangential half lightlike submanifold $M$ of an indefinite Sasakian manifold $\bar{M}$.

Theorem 3.2. Let $M$ be a tangential half lightlike submanifold of an indefinite Sasakian manifold $\bar{M}$. Then the vector field $\zeta$ is conjugate to the lightlike vector field $J \xi$. Moreover, $\zeta$ is an asymptotic vector field.

Proof. Applying $\bar{\nabla}_{X}$ to $g(\zeta, \xi)=\bar{g}(\zeta, L)=0$ and using (2.1), (2.2), (2.6), (2.8), (2.10), (2.11) and (2.16), we have

$$
B(X, \zeta)=-g(X, J \xi), D(X, \zeta)=-\epsilon g(X, J L), \forall X \in \Gamma(T M),
$$

respectively. Consequently we get $B(\zeta, J \xi)=D(X, J \xi)=0$. Thus $\zeta$ is conjugate to $J \xi$. From $(3.3)$ we also have $B(\zeta, \zeta)=D(\zeta, \zeta)=0$ and $h(\zeta, \zeta)=0$ due to $(2.1)$. Thus $\zeta$ is an asymptotic vector field.

Note 1. If $\zeta$ is tangent to $M$, then $\zeta$ does not belong to $\operatorname{Rad}(T M)$ by Theorem 3.1. This enables one to choose a screen distribution $S(T M)$ which contains $\zeta$. This fact implies that if $\zeta$ is tangent to $M$, then it belongs to $S(T M)$. Călin also proved this result [2] which Kang et al. [12] and Duggal-Sahin [7, 8] assumed in their papers. Although, in general, $S(T M)$ is not unique, it is canonically isomorphic to the factor vector bundle $S(T M)^{*}=T M / \operatorname{Rad} T M$ considered by Kupeli [13]. Thus all $S(T M)$ are mutually isomorphic. For this reason, in the rest of this section, let $M$ be a tangential half lightlike submanifold equipped with a screen distribution $S(T M)$ which contains $\zeta$. 
Definition. We say that $M$ is an CR-submanifold [1] of $\bar{M}$ if $M$ is endow with two complementary distributions $\mathcal{D}$ and $\mathcal{D}^{\perp}$ of $M$ such that

$$
J(\mathcal{D})=\mathcal{D}, \quad J\left(\mathcal{D}^{\perp}\right) \subset T M^{\perp} .
$$

Theorem 3.3. Every tangential half lightlike submanifold $M$ of an indefinite Sasakian manifold $\bar{M}$ is an indefinite CR-submanifold of $\bar{M}$.

Proof. Since $\zeta$ belongs to $S(T M), J \xi$ and $J N$ are lightlike vector fields such that $g(J \xi, J N)=1$ and $J(\operatorname{Rad}(T M)) \oplus J(\operatorname{ltr}(T M))$ is a distribution of $M$, of rank 2. Thus the screen distribution $S(T M)$ splits as follows:

$$
S(T M)=\{J(\operatorname{Rad}(T M)) \oplus J(\operatorname{ltr}(T M))\} \oplus_{\text {orth }} J\left(S\left(T M^{\perp}\right)\right) \oplus_{\text {orth }} H_{o},
$$

where $H_{o}$ is an almost complex distribution on $M$ with respect to $J$, i.e., $J\left(H_{o}\right)=H_{o}$. Thus the general decompositions (2.4) and (2.5) reduce to

$$
T M=H \oplus H^{\prime}, \quad T \bar{M}=H \oplus H^{\prime} \oplus \operatorname{tr}(T M),
$$

where $H$ and $H^{\prime}$ are 2- and 1-lightlike distributions on $M$ such that

$$
\begin{aligned}
& H=\operatorname{Rad}(T M) \oplus_{\text {orth }} J(\operatorname{Rad}(T M)) \oplus_{\text {orth }} H_{o}, \\
& H^{\prime}=J(\operatorname{ltr}(T M)) \oplus_{\text {orth }} J\left(S\left(T M^{\perp}\right)\right) .
\end{aligned}
$$

By direct calculations we obtain $J(H)=H$ and $J\left(H^{\prime}\right) \subset T M^{\perp}$. Thus $M$ is an indefinite CR-submanifold of $\bar{M}$.

Consider the local lightlike vector fields $U$ and $V$ and the local non-lightlike vector field $W$ on $S(T M)$, of sign $\epsilon$, such that

$$
U=-J N, \quad V=-J \xi, \quad W=-J L .
$$

Denote by $S$ the projection morphism of $T M$ on $H$ with respect to the decomposition $(3.4)_{1}$. Then any vector field on $M$ is expressed as follows

$$
X=S X+u(X) U+w(X) W, \quad J X=F X+u(X) N+w(X) L,
$$

where $u, v$ and $w$ are 1-forms locally defined on $M$ by

$$
u(X)=g(X, V), \quad v(X)=g(X, U), \quad w(X)=\epsilon g(X, W)
$$

and $F$ is a tensor field of type $(1,1)$ globally defined on $M$ by

$$
F X=J S X, \quad \forall X \in \Gamma(T M) .
$$

Applying $\bar{\nabla}_{X}$ to $(3.5)$ and $(3.6)_{2}$ and using $(2.3),(2.6) \sim(2.11)$ and (3.6), for all $X, Y \in \Gamma(T M)$, we have

(3.10) $\nabla_{X} V=F\left(A_{\xi}^{*} X\right)-\tau(X) V-\epsilon \phi(X) W$,

(3.11) $\nabla_{X} W=F\left(A_{L} X\right)+\phi(X) U$,

(3.12) $\left(\nabla_{X} F\right)(Y)=u(Y) A_{N} X+w(Y) A_{L} X-B(X, Y) U-D(X, Y) W$ $+\theta(Y) X-g(X, Y) \zeta$. 
Theorem 3.4. Let $M$ be a tangential half lightlike submanifold of an indefinite Sasakian manifold $\bar{M}$. Then $F$ is parallel on $H$ with respect to the induced connection $\nabla$ if and only if $H$ is parallel with respect to $\nabla$.

Proof. Taking $Y \in \Gamma(H)$, we have $F Y=J Y \in \Gamma(H)$. Applying $J$ to (2.6) with $Y \in \Gamma(H)$ and using (2.3), (2.6), (3.5) and (3.6) $)_{2}$, we have

$$
\begin{aligned}
& B(X, F Y)=g\left(\nabla_{X} Y, V\right), \quad D(X, F Y)=\epsilon g\left(\nabla_{X} Y, W\right) \\
& \left(\nabla_{X} F\right)(Y)=-B(X, Y) U-D(X, Y) W+\theta(Y) X-g(X, Y) \zeta
\end{aligned}
$$

Assume that $F$ is parallel on $H$ with respect to the connection $\nabla$. For any $X, Y \in \Gamma(H)$, we have $\left(\nabla_{X} F\right) Y=0$. Taking the scalar product with $V$ and $W$ to (3.14) with $\left(\nabla_{X} F\right) Y=0$ and using the facts $u(Y)=w(Y)=0$ and $g(\zeta, V)=-\theta(J \xi)=0, g(\zeta, W)=-\theta(J L)=0$ due to (2.1), we show that $B(X, Y)=0$ and $D(X, Y)=0$ for all $X, Y \in \Gamma(H)$, respectively. From (3.13), we have $g\left(\nabla_{X} Y, V\right)=0$ and $g\left(\nabla_{X} Y, W\right)=0$. This imply $\nabla_{X} Y \in \Gamma(H)$ for all $X, Y \in \Gamma(H)$. Thus $H$ is parallel with respect to $\nabla$.

Conversely if $H$ is parallel with respect to $\nabla$, from (3.13) we have

$$
B(X, F Y)=0, D(X, F Y)=0, \forall X, Y \in \Gamma(H) .
$$

For any $Y \in \Gamma(H)$, we show that $F^{2} Y=J^{2} Y=-Y+\theta(Y) \zeta$. By the proof of Theorem 3.1, we show that $B(X, \zeta)=g(X, V)=u(X)=0$ and $D(X, \zeta)=$ $\epsilon g(X, W)=w(X)=0$ for all $X \in \Gamma(T M)$. Replacing $Y$ by $F Y$ to (3.15), we have $B(X, Y)=0$ and $D(X, Y)=0$ for any $X, Y \in \Gamma(H)$. By this results and (3.14) we show that $F$ is parallel on $H$ with respect to $\nabla$.

Theorem 3.5. Let $M$ be a tangential lightlike submanifold of an indefinite Sasakian manifold $\bar{M}$. If $F$ is parallel with respect to $\nabla$, then $H$ and $H^{\prime}$ are integrable and parallel distributions with respect to $\nabla$ and $M$ is locally a product manifold $M_{2} \times M^{\sharp}$, where $M_{2}$ is a leaf of $H^{\prime}$ and $M^{\sharp}$ is a leaf of $H$.

Proof. Assume that $F$ is parallel on $M$ with respect to the induced connection $\nabla$. Then $F$ is parallel on $H$ with respect to $\nabla$. By Theorem 3.4, $H$ is a parallel distribution on $M$. Applying $F$ to $(3.12)$ with $\left(\nabla_{X} F\right) Y=0$, we have

$$
u(Y) F\left(A_{N} X\right)+w(Y) F\left(A_{L} X\right)+\theta(Y) F X=0
$$

due to $F U=F W=F \zeta=0$. Replacing $Y$ by $U$ and $W$ to this by turns and using (3.7), we have $F\left(A_{N} X\right)=0$ and $F\left(A_{L} X\right)=0$. Taking the scalar product with $W$ and $N$ to $(3.12)$ with $\left(\bar{\nabla}_{X} F\right) Y=0$ by turns, we have

$$
\begin{aligned}
& D(X, Y)=u(Y) w\left(A_{N} X\right)+w(Y) w\left(A_{L} X\right)+\theta(Y) w(X), \\
& w(Y) g\left(A_{L} X, N\right)+\theta(Y) \eta(X)=0, \quad \forall X, Y \in \Gamma(T M) .
\end{aligned}
$$

Replacing $Y$ by $\xi$ to (3.16), we get $\phi(X)=0$. Also replacing $Y$ by $W$ to (3.17), we have $\rho(X)=0$. From this results, (3.9) and (3.11), we get $\nabla_{X} U=\tau(X) U$ and $\nabla_{X} W=0$ for all $X \in \Gamma\left(H^{\prime}\right)$. Thus $H^{\prime}$ is also a parallel distribution of $M$. By the decomposition theorem of de Rham [3], we have $M_{2} \times M^{\sharp}$, where $M_{2}$ is a leaf of $H^{\prime}$ and $M^{\sharp}$ is a leaf of $H$. 


\section{Ascreen half lightlike submanifolds}

Definition. A half lightlike submanifold $M$ of an indefinite Sasakian manifold $\bar{M}$ is said to be ascreen if $\zeta$ belongs to $\operatorname{Rad}(T M) \oplus \operatorname{ltr}(T M)$.

For any ascreen half lightlike submanifold $M, \zeta$ is decomposed as

$$
\zeta=a \xi+b N,
$$

where $a=\theta(N)$ and $b=\theta(\xi)$ are smooth functions on $\bar{M}$. As $\bar{g}(\zeta, \zeta)=1$, we get $2 a b=1$. This implies that $a \neq 0$ and $b \neq 0$. Now we may assume that $\epsilon=1$. Then the normal vector field $L$ is a unit spacelike one on $S\left(T M^{\perp}\right)$.

Lemma 4.1. Let $M$ be a half lightlike submanifold of an indefinite Sasakian manifold $\bar{M}$. Then $M$ is an ascreen half lightlike submanifold of $\bar{M}$ if and only if $J(\operatorname{Rad}(T M))=J(\operatorname{ltr}(T M))$.

Proof. $(\Rightarrow)$ Assume that $\zeta=a \xi+b N$. Applying $J$ to this and using $J \zeta=0$, we get $J \xi=k J N$, where $k$ is a non-vanishing smooth function on $\bar{M}$ such that $k=-\frac{b}{a}$. This fact implies $J(\operatorname{Rad}(T M)) \cap J(\operatorname{ltr}(T M)) \neq\{0\}$. As both have rank 1 , we get $J(\operatorname{Rad}(T M))=J(\operatorname{ltr}(T M))$.

$(\Leftarrow)$ Assume that $J(\operatorname{Rad}(T M))=J(\operatorname{ltr}(T M))$. Then there exists a nonvanishing smooth real valued function $k$ such that $J \xi=k J N$. Taking the scalar product with $J \xi$ and $J N$ in this equation by turns, we get $b^{2}=k(a b-1)$ and $k a^{2}=a b-1$, respectively. From these two equations we have $a \neq 0 ; b \neq 0$ and $b^{2}=(k a)^{2}$. The last equation implies $b=k a$ or $b=-k a$. If $b=k a$, then we have $a b=k a^{2}=a b-1$. It is a contradiction. Thus we have $b=-k a$. In this case we get $2 a b=1$. Since $k=-\frac{b}{a}, a \neq 0$ and $J \xi=k J N$, we see that $a J \xi+b J N=0$. Applying $J$ to this equation and using (2.1), we have $2 a b \zeta=a \xi+b N$. From the fact $2 a b=1$, we get $\zeta=a \xi+b N$.

Theorem 4.2. (1) There exists no screen conformal ascreen half lightlike submanifold $M$ of an indefinite Sasakian manifold $\bar{M}$.

(2) There exists no ascreen half lightlike submanifold $M$ of an indefinite Sasakian manifold $\bar{M}$ such that $S(T M)$ is totally umbilical in $M$.

(3) There exists no proper totally umbilical ascreen half lightlike submanifold $M$ of an indefinite Sasakian manifold $\bar{M}$.

Proof. Applying $\bar{\nabla}_{X}$ to $\bar{g}(J \xi, N)=0, \bar{g}(J \xi, L)=0$ and $\bar{g}(J N, L)=0$ and using $(2.3),(2.6) \sim(2.11),(2.13) \sim(2.15)$, we have

$$
\begin{aligned}
& b \eta(X)+B(X, J N)=C(X, J \xi), \forall X \in \Gamma(T M), \\
& B(X, J L)=D(X, J \xi), C(X, J L)=D(X, J N) .
\end{aligned}
$$

Replacing $X$ by $\xi$ to (4.2) and using the first equation of (2.11), we have

$$
b=C(\xi, J \xi) .
$$

(1) If $M$ is screen conformal, then, from (4.4), we have

$$
b=C(\xi, J \xi)=\varphi B(\xi, J \xi)=\varphi 0=0 .
$$


It is a contradiction to $b \neq 0$. Thus there exists no screen conformal ascreen half lightlike submanifold $M$ of an indefinite Sasakian manifold $\bar{M}$.

(2) If $S(T M)$ is totally umbilical in $M$, then we have

$$
b=C(\xi, J \xi)=\gamma g(\xi, J \xi)=\gamma 0=0 .
$$

This also a contradiction to $b \neq 0$. Thus there exists no ascreen half lightlike submanifold $M$ of $\bar{M}$ such that $S(T M)$ is totally umbilical in $M$.

(3) If $M$ is totally umbilical, then, from $(4.3)_{1}$, we have

$$
\beta g(X, J L)=\delta g(X, J \xi), \forall X \in \Gamma(T M) .
$$

Replacing $X$ by $J L$ in (4.5) and using the facts $g(J L, J L)=1$ and $g(J L, J \xi)=$ 0 , we have $\beta=0$. Replacing $X$ by $J \xi$ and $J N$ by turns to (4.5) with $\beta=0$, we have $\delta=0$. This results imply that $M$ is totally geodesic. Thus there exists no proper totally umbilical ascreen half lightlike submanifold $M$ of $\bar{M}$.

Corollary 4.3. Every totally umbilical ascreen half lightlike submanifold $M$ of an indefinite Sasakian manifold $\bar{M}$ is totally geodesic.

Definition. Let $M$ be a lightlike submanifold of $\bar{M}$. We say that $M$ is a $C R$ lightlike submanifold [4] if the following two conditions are fulfilled:

(A) $J(\operatorname{Rad}(T M))$ is a distribution on $M$ such that

$$
\operatorname{Rad}(T M) \cap J(\operatorname{Rad}(T M))=\{0\} .
$$

(B) There exists vector bundles $H_{o}$ and $H^{\prime}$ over $M$ such that

$S(T M)=\left\{J(\operatorname{Rad}(T M)) \oplus H^{\prime}\right\} \oplus_{\text {orth }} H_{o} ; J\left(H_{o}\right)=H_{o} ; J\left(H^{\prime}\right)=K_{1} \oplus_{\text {orth }} K_{2}$, where $H_{o}$ is a non-degenerate almost complex distribution on $M$, and $K_{1}$ and $K_{2}$ are vector subbundles of $l \operatorname{tr}(T M)$ and $S\left(T M^{\perp}\right)$, respectively.

Theorem 4.4. Every ascreen half lightlike submanifold $M$ of an indefinite Sasakian manifold $\bar{M}$ is a CR-lightlike submanifold of $\bar{M}$.

Proof. From Lemma 4.1, we shown that $\operatorname{Rad}(T M) \cap J(\operatorname{Rad}(T M))=\{0\}$. Using Lemmas 4.1 and 4.2, the screen $S(T M)$ is decomposed as follow:

$$
S(T M)=J(\operatorname{Rad}(T M)) \oplus_{\text {orth }} J\left(S\left(T M^{\perp}\right)\right) \oplus_{\text {orth }} H_{c},
$$

where $H_{c}$ is a non-degenerate and almost complex distribution on $M$ with respect to $J$. Let $H_{o}=H_{c}$ and $H^{\prime}=J\left(S\left(T M^{\perp}\right)\right)$. By direct calculations we obtain $J\left(H_{c}\right)=H_{c}$ and $J\left(H^{\prime}\right)=K_{1} \oplus_{\text {orth }} K_{2}$ where $K_{1}=\{0\}$ and $K_{2}=$ $S\left(T M^{\perp}\right)$. Thus $M$ is a CR-submanifold of $\bar{M}$.

Theorem 4.5. Let $M$ be an ascreen half lightlike submanifold of an indefinite Sasakian manifold $\bar{M}$ such that $\operatorname{dim} \bar{M}>4$. Then $S(T M)$ is not integrable.

Proof. Consider two local vector fields $V_{c}$ and $W_{c}$ on $S(T M)$ defined by

$$
V_{c}=-b^{-1} J \xi=a^{-1} J N, \quad W_{c}=-J L .
$$


Then $V_{c}$ is a unit timelike vector field on $S(T M)$ and $W_{c}$ is a unit spacelike vector field on $S(T M)$. Applying the operator $J$ to the equation $V_{c}=-b^{-1} J \xi$ and using the first equation of (2.1) and $2 a b=1$, we get

$$
J V_{c}=a \xi-b N .
$$

Denote by $S_{c}$ the projection morphism of $T M$ on $H_{c}$ with respect to the decomposition (4.6). Then any vector field $X$ on $M$ is expressed as follows

$$
X=S_{c} X+\eta(X) \xi+v_{c}(X) V_{c}+w_{c}(X) W_{c},
$$

where $v_{c}$ and $w_{c}$ are 1-forms locally defined on $M$ by

$$
v_{c}(X)=-g\left(X, V_{c}\right), w_{c}(X)=g\left(X, W_{c}\right), \forall X \in \Gamma(T M) .
$$

Applying $J$ to (4.8) and using (4.7) and the fact $\theta(X)=b \eta(X)$, we have

$$
J X=F_{c} X+a v_{c}(X) \xi-\theta(X) V_{c}-b v_{c}(X) N+w_{c}(X) L,
$$

where $F_{c}$ is a tensor field of type $(1,1)$ globally defined on $M$ by

$$
F_{c} X=J S_{c} X, \quad \forall X \in \Gamma(T M) .
$$

Applying $J$ to (4.10) and using (2.1), (4.7) and the fact $\theta(X)=b \eta(X)$, we have

$$
F_{c}^{2} X=-X+\eta(X) \xi+v_{c}(X) V_{c}+w_{c}(X) W_{c} .
$$

For any $X, Y \in \Gamma(S(T M))$, by $(2.9)$ we have $C(X, Y)=g\left(\nabla_{X} Y, N\right)$. Thus

$$
C(X, Y)-C(Y, X)=g\left(\nabla_{X} Y-\nabla_{Y} X, N\right)=\eta([X, Y]) .
$$

If $S(T M)$ is integrable, then $\eta([X, Y])=0$ for all $X, Y \in \Gamma(S(T M))$. This implies that $C$ is symmetric on $S(T M)$. Applying $\bar{\nabla}_{X}$ to $\bar{g}(\zeta, Y)=0$ and using (2.2), (2.6) and (2.9), we have

$$
\bar{g}(J X, Y)+b C(X, Y)+a B(X, Y)=0, \quad \forall X \in \Gamma(T M) .
$$

Using this and the fact $B$ and $C$ are symmetric, we get

$$
\bar{g}(J X, Y)-\bar{g}(X, J Y)=0, \quad \forall X, Y \in \Gamma(S(T M)) .
$$

Using (2.1), we show that the equation $\bar{g}(X, J Y)+\bar{g}(J X, Y)=0$ is equivalent to the equation $\bar{g}(J X, J Y)=g(X, Y)-\theta(X) \theta(Y)$. Thus we have $\bar{g}(J X, Y)=0$ for all $X, Y \in \Gamma(S(T M))$. From this, (4.1) and (4.10), we have

$$
g(F X, Y)=0, \quad \forall X, Y \in \Gamma(S(T M) .
$$

As $S(T M)$ is non-degenerate, we have $F X=0$ for all $X \in \Gamma(S(T M))$. From this and (4.11), we have $X=0$ for all $X \in \Gamma(S(T M))$, i.e., $S(T M)=\{0\}$. It is a contradiction as $\operatorname{dim} \bar{M}>4$. Thus $S(T M)$ is not integrable.

Definition. We say that $M$ is locally symmetric [9] if its curvature tensor $R$ be parallel, i.e., have vanishing covariant differential, $\nabla R=0$.

Theorem 4.6. Let $M$ be a totally umbilical ascreen half lightlike submanifold of an indefinite Sasakian manifold $\bar{M}$. If $M$ is locally symmetric space, then $M$ is a space of constant curvature 1 . 
Proof. Substituting (4.1) into (2.2) and using (2.6), (2.7), (2.10), (2.11), we get

$$
\begin{aligned}
J X= & -a A_{\xi}^{*} X-b A_{N} X+\{X a-a \tau(X)\} \xi \\
& +\{X b+b \tau(X)\} N-\{a \phi(X)-b \rho(X)\} L, \forall X \in \Gamma(T M) .
\end{aligned}
$$

Comparing (4.10) and (4.12), for all $X \in \Gamma(T M)$, we have

(4.13) $X a-a \tau(X)=a v_{c}(X), \quad X b+b \tau(X)=-b v_{c}(X)$,

(4.14) $a A_{\xi}^{*} X+b A_{N} X=\theta(X) V_{c}-F_{c} X, w_{c}(X)=-a \phi(X)+b \rho(X)$.

Applying $\bar{\nabla}_{X}$ to $b V_{c}=-J \xi$ with $X \in \Gamma(T M)$ and using (2.3), (2.6), (2.9), (2.10), (4.10) and (4.13), we get

$$
b \nabla_{X} V_{c}=-b X+b v_{c}(X) V_{c}-\phi(X) W_{c}+a v_{c}\left(A_{\xi}^{*} X\right) \xi+F\left(A_{\xi}^{*} X\right) .
$$

Assume that $M$ is a totally umbilical ascreen half lightlike submanifold of $\bar{M}$. By Corollary 4.3, $M$ is totally geodesic. Thus we have $B=D=\phi=A_{\xi}^{*}=0$ due to (2.11) and (2.13). Also we have $A_{L} X=\rho(X) \xi$ for all $X \in \Gamma(T M)$ due to $(2.15)$. Since $b \neq 0,(4.15)$ reduce to

$$
\nabla_{X} V_{c}=-X+v_{c}(X) V_{c}, \forall X \in \Gamma(T M) .
$$

Using (4.16) and the fact that $\nabla$ is a torsion free connection, we have

$$
R(X, Y) V_{c}=2 d v_{c}(X, Y) V_{c}+v_{c}(X) Y-v_{c}(Y) X, \forall X, Y \in \Gamma(T M) .
$$

Taking the scalar product with $V_{c}$ in this equation and using $(4.9)_{1}$, we have $d v_{c}=0$. From this fact we deduce the following equation:

$$
R(X, Y) V_{c}=v_{c}(X) Y-v_{c}(Y) X, \forall X, Y \in \Gamma(T M) .
$$

Differentiating (4.9) with $Y \in \Gamma(T M)$ and using (4.9) and (4.16), we have

$$
\left(\nabla_{X} v_{c}\right)(Y)=g(X, Y)+v_{c}(X) v_{c}(Y), \quad \forall X, Y \in \Gamma(T M) \text {. }
$$

Differentiating (4.17) with $Z \in \Gamma(T M)$ and using (4.17) and the fact that $M$ is locally symmetric, i.e., $\nabla_{X} R=0$, we have

$$
R(X, Y) \nabla_{Z} V_{c}=\left(\nabla_{X} v_{c}\right)(X) Y-\left(\nabla_{Z} v_{c}\right)(Y) X, \forall X, Y \in \Gamma(T M) .
$$

Substituting (4.16) and (4.18) in this equation and using (4.17), we obtain

$$
R(X, Y) Z=g(Y, Z) X-g(X, Z) Y, \quad \forall X, Y \in \Gamma(T M) .
$$

From this equation and (2.17), we deduce our theorem.

The induced Ricci type tensor $R^{(0,2)}$ of $M$ is defined by

$$
R^{(0,2)}(X, Y)=\operatorname{trace}\{Z \rightarrow R(Z, X) Y\}, \forall X, Y \in \Gamma(T M) .
$$

In general, the induced Ricci type tensor $R^{(0,2)}$ is not symmetric $[4,5,6]$. The Ricci type tenor field $R^{(0,2)}$ of $M$ is called its induced Ricci tensor [6] of $M$ if it is symmetric. A symmetric $R^{(0,2)}$ tensor will be denoted by Ric. Note that $R^{(0,2)}$ is the induced Ricci tensor of $M$ if and only if the 1-form $\tau$ given by (2.7) is closed, i.e., $d \tau=0$, on any $\mathcal{U} \subset M[5]$. 
Definition. We define a connection $\nabla^{\ell}$ on the lightlike transversal vector bundle $\operatorname{ltr}(T M)$ by $\nabla_{X}^{\ell} N=\tau(X) N$ for all $X \in \Gamma(T M)$. We say that $\nabla^{\ell}$ is the lightlike transversal connection of $M$. We define a curvature tensor $R^{\ell}$ of the lightlike transversal vector bundle $\operatorname{ltr}(T M)$ by

$$
R^{\ell}(X, Y) N=\nabla_{X}^{\ell} \nabla_{Y}^{\ell} N-\nabla_{Y}^{\ell} \nabla_{X}^{\ell} N-\nabla_{[X, Y]}^{\ell} N
$$

for all $X, Y \in \Gamma(T M)$. If $R^{\ell}$ vanishes identically, then the lightlike transversal connection $\nabla^{\ell}$ of $M$ is said to be flat (or trivial) [10, 11].

Theorem 4.7 ([10, 11]). Let $M$ be a half lightlike submanifold of a semiRiemannian manifold $\bar{M}$. The following assertions are equivalent:

(1) The lightlike transversal connection of $M$ is flat, i.e., $R^{\ell}=0$.

(2) The 1-form $\tau$ is closed, i.e., $d \tau=0$, on any $\mathcal{U} \subset M$.

(3) The Ricci type tensor $R^{(0,2)}$ is an induced Ricci tensor of $M$.

Proof. Applying the operator $\nabla_{X}^{\ell}$ to $\nabla_{Y} N=\tau(Y) N$, we have

$$
\nabla_{X}^{\ell} \nabla_{Y}^{\ell} N=X(\tau(Y)) N+\tau(X) \tau(Y) N .
$$

Thus we have $R^{\ell}(X, Y) N=2 d \tau(X, Y) N$ for all $X, Y \in \Gamma(T M)$. From this we have our assertion.

Theorem 4.8. Let $M$ be a locally symmetric totally umbilical ascreen half lightlike submanifold of an indefinite Sasakian manifold $\bar{M}$. Then the lightlike transversal connection of $M$ is flat and $R^{(0,2)}$ is an induced Ricci tensor of $M$.

Proof. Using (2.6), (2.7) and (2.8), we have

$$
\begin{aligned}
& \bar{R}(X, Y) N=-\nabla_{X}\left(A_{N} Y\right)+\nabla_{Y}\left(A_{N} X\right)+A_{N}[X, Y] \\
+ & \tau(X) A_{N} Y-\tau(Y) A_{N} X+\rho(X) A_{L} Y-\rho(Y) A_{L} X \\
+ & \left\{B\left(Y, A_{N} X\right)-B\left(X, A_{N} Y\right)+2 d \tau(X, Y)+\phi(X) \rho(Y)-\phi(Y) \rho(X)\right\} N \\
+ & \left\{D\left(Y, A_{N} X\right)-D\left(X, A_{N} Y\right)+2 d \rho(X, Y)+\rho(X) \tau(Y)-\rho(Y) \tau(X)\right\} L .
\end{aligned}
$$

Taking the scalar product with $\xi$ to $(4.20)$ and using $B=\phi=0$, we have

$$
\bar{g}(\bar{R}(X, Y) N, \xi)=2 d \tau(X, Y), \forall X, Y \in \Gamma(T M) .
$$

Using (4.7), (4.19), (4.21) and the fact $\bar{R}(X, Y) Z=R(X, Y) Z$, we have

$$
\begin{aligned}
2 d \tau(X, Y) & =\bar{g}(\bar{R}(X, Y) N, \xi)=-\bar{g}(\bar{R}(X, Y) \xi, N) \\
& =-\bar{g}(R(X, Y) \xi, N)=g(X, \xi) \eta(Y)-g(Y, \xi) \eta(X)=0
\end{aligned}
$$

for all $X, Y \in \Gamma(T M)$. Thus, from Theorem 4.7, we have our theorem.

\section{References}

[1] A. Bejancu, Geometry of CR-submanifolds, D. Reil, Dordrecht, 1986.

[2] C. Călin, Contributions to geometry of CR-submanifold, Thesis, University of Iasi (Romania), 1998.

[3] G. de Rham, Sur la réductibilité d'un espace de Riemannian, Comm. Math. Helv. 26 (1952), 328-344. 
[4] K. L. Duggal and A. Bejancu, Lightlike Submanifolds of Semi-Riemannian Manifolds and Applications, Kluwer Acad. Publishers, Dordrecht, 1996.

[5] K. L. Duggal and D. H. Jin, Half-Lightlike Submanifolds of Codimension 2, Math. J. Toyama Univ. 22 (1999), 121-161.

[6] _ Null curves and Hypersurfaces of Semi-Riemannian Manifolds, World Scientific, 2007.

[7] K. L. Duggal and B. Sahin, Generalized Cauchy-Riemann lightlike submanifolds of Kaehler manifolds, Acta Math. Hungar. 112 (2006), no. 1-2, 107130.

[8] _ Lightlike Submanifolds of indefinite Sasakian manifolds, Int. J. Math. Math. Sci. 2007 (2007), Art. ID 57585, 21 pp.

[9] D. H. Jin, Geometry of lightlike hypersurfaces of an indefinite Sasakian manifold, Indian J. Pure Appl. Math. 41 (2010), no. 4, 569-581.

[10] _ Transversal half lightlike submanifolds of an indefinite Sasakian manifold, J. Korea Soc. Math. Educ. Ser. B Pure Appl. Math. 18 (2011), no. 1, 51-62.

[11] . Half lightlike submanifolds of an indefinite Sasakian manifold, J. Korea Soc. Math. Educ. Ser. B Pure Appl. Math. 18 (2011), no. 2, 173-183.

[12] T. H. Kang, S. D. Jung, B. H. Kim, H. K. Pak, and J. S. Pak, Lightlike hypersurfaces of indefinite Sasakian manifolds, Indian J. Pure Appl. Math. 34 (2003), no. 9, 1369-1380.

[13] D. N. Kupeli, Singular Semi-Riemannian Geometry, Mathematics and Its Applications, Kluwer Acad. Publishers, Dordrecht, 1996.

Department of Mathematics

DongGuK UNIVERSITY

KYONGJU 780-714, Korea

E-mail address: jindh@dongguk.ac.kr 